\title{
Experimentally Observed Instability of a Laminar Ekman Flow in a Rotating Basin ${ }^{1,2}$
}

\author{
By A. B. ARONS, A. P. INGERSOLL, T. GREEN III \\ Amherst College and Woods Hole Oceanographic Institution
}

(Manuscript received August I8, I960)

\begin{abstract}
In studying the axi-symmetric flow induced by source-sink distributions in a rotating cylindrical basin in the absence of radial barriers, a highly organized pattern of instability has been observed in the laminar Ekman layer along the bottom of the basin. The instability manifests itself in the form of almost perfectly concentric cylindrical sheets or curtains of water which rise as sharply defined vertical jets from the Ekman layer and penetrate the entire depth of fluid. A less sharply defined downward motion between the curtains completes the circulation celis thus developed. At some maximum critical radius, the curtains usually disappear, and the flow at larger radii is a stable, laminar Ekman flow. Quantitative observations of ring spacing and critical radius are reported for experiments in which angular velocity, flow rate, viscosity and total depth of water were varied over experimentally available ranges.
\end{abstract}

\section{Introduction}

It has been shown in an earlier paper in this journal (Stommel, Arons, and Faller, 1959) that it is possible to drive systematic circulations in a rotating basin by means of suitably distributed sources and sinks of fluid. This technique offers the possibility of studying flows in a homogenous system, without the complexities introduced by density gradients and convection phenomena in the presence of temperature fields.

In studying the axi-symmetric flows induced by source-sink distributions in an "open" cylindrical basin (i. e. in the absence of radial barriers), we have observed a highly organized pattern of instablity in the laminar Ekman flow which forms a boundary layer in contact with the bottom of the basin. It is within this Ek-

\footnotetext{
1 Contribution No. II 35 of the Woods Hole Oceanographic Institution.

2 The rotating tank facility was constructed and operated under a grant from the Research Corporation,

Tellus XIII (1961), 1
}

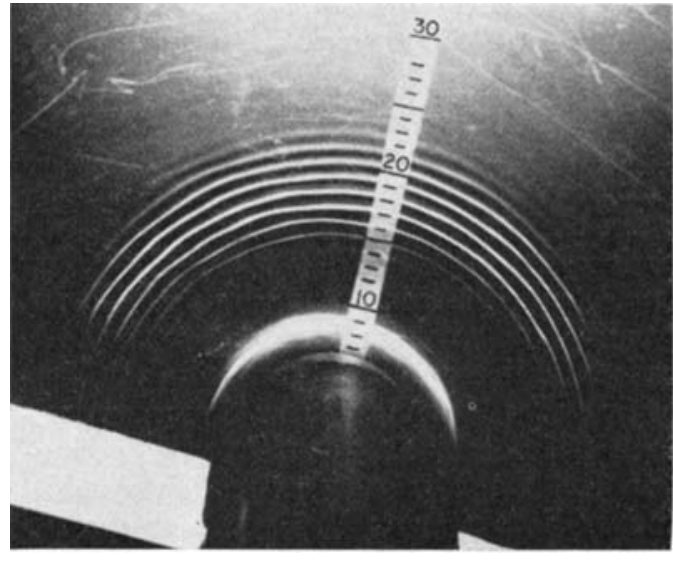

Fig. I. Top view of tank showing concentric curtains of fluorescein dyed fluid rising from Ekman layer. Source at center of tank near bottom of photograph; rotation counter clockwise; relative zonal velocity $\nu_{\phi}$ clockwise; Ekman layer spreading radially outwards. (Scale markings in $\mathrm{cm}$.) 


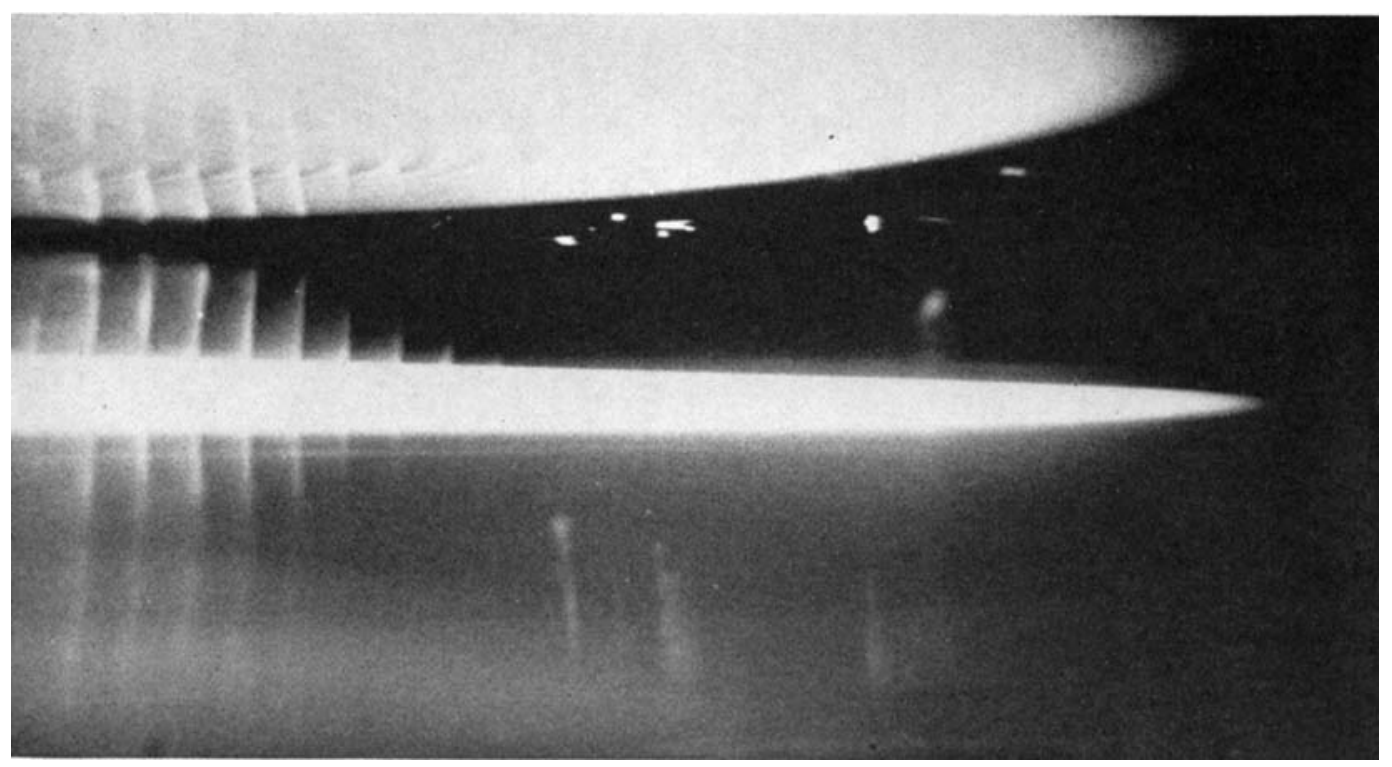

Fig. 2. Side view through wall of tank, showing sharpness of rising curtains and Ekman layer along bottom. Center of tank is to left of photograph; dyed region spread from left to right. Jets at right look lower than those at left because upward velocities are smaller at radii approaching the critical radius. Multiple images are present because of reflections at bottom and at water surface, i.e. colored region, circles, and downward jets appearing in upper part of photograph are surface reflections of the interior flow. Upward velocity of curtains is about $0.7 \mathrm{~cm} / \mathrm{min}$.

man layer that fluid is transported radially, either inward or outward, across isobars of the essentially geostrophic bulk flow in the interior of the basin.

The instability manifests itself in the form of almost perfectly concentric cylindrical sheets or curtains of fluid which rise as sharply defined vertical jets from the Ekman layer and penetrate the entire depth of fluid (Figures I, 2) forming circulation cells with less sharply defined downward motions in the regions between the upward rising curtains. The radial spacing between the rising curtains is highly regular and is a function of angular velocity, viscosity, and total depth of fluid in the tank, i.e. it is indicated to be a function of the Taylor or of the Ekman number. At some maximum critical radius the curtains usually disappear, and the flow at larger radii is a stable, laminar Ekman flow; this points to the existence of a critical Reynolds number for the development and maintenance of the instability.

STERN (1960) has examined, theoretically, the stability of Ekman flows in a somewhat simpler geometry. His analysis predicts, at least qualitatively, the principal features of the instability we describe in this paper.

\section{Method of observation}

The experimental system consists of a cylindrical lucite tank, $130 \mathrm{~cm}$ diameter and about $30 \mathrm{~cm}$ deep, mounted on a carefully levelled rotating platform, driven by a synchronous motor through a Graham variable speed transmission. During experiments, a transparent cover was placed over the tank to prevent the development of "wind driven" circulations. The "source" consisted of a measured flow of water supplied to a funnel mounted at the center of the tank. To reduce turbulence in the source region, this water entered a vertical cylinder with a diameter of twelve centimeters, mounted at the center of the basin in such a way that the source water could run radially outward around the bottom of the cylinder.

The flow within the system was made visible by using potassium permanganate or fluorescein dye in the source water. Photographs could be taken from above or through the side of the tank. Observations of the velocity of the zonal geostrophic flow in the interior of the basin were made by periodically photographing the displacement of small particles or bits 
of paper moving with the water (at the surface).

Thermal circulations were minimized by performing experiments when tank water and source water were in equilibrium with room temperature. The latter was changed in order to change the temperature (and, therefore, the viscosity of the water) in certain series of experiments. Check runs at zero source strength showed no systematic circulations of the kind observed at non-zero source strengths; no local velocity noted at zero source strength exceeded $0.09 \mathrm{~cm} / \mathrm{sec}$.

In several runs, where the temperature of the source water was $\mathrm{I}-2^{\circ} \mathrm{C}$ lower than that of the water in the tank, rings were observed as usual; in other words, the pattern occurred in spite of the thermal stability induced by the denser Ekman layer water.

Experiments were performed at angular velocities of $\mathrm{I}$ to 2 radians/sec., source strength of I to $5 \mathrm{~cm}^{3} / \mathrm{sec}$., kinematic viscosities in the range of 0.007 to $0.012 \mathrm{~cm}^{2} / \mathrm{sec}$, and water depths of about 2 to $12 \mathrm{~cm}$. The laminar Ekman layer under these circumstances has a depth of the order of $\mathrm{I} \mathrm{mm}$.

In all cases, quantitative observations were made only after the system had had a period of the order of one hour in which to arrive at a steady state.

\section{Equations of the basic flow}

Using cylindrical coordinates $r, \phi, z$ and taking $\phi$ positive in the same sense as $\omega$, the angular velocity of the tank, we have:

For the depth of water when the tank is rotating in static equilibrium:

$$
\begin{aligned}
h & =h_{0}+\frac{\omega^{2} r^{2}}{2 g} \\
& =D_{0}-\frac{\omega^{2}}{2 g}\left(\frac{a^{2}}{2}-r^{2}\right)
\end{aligned}
$$

where $h_{0}$ is the depth at $r=0 ; D_{0}$ is the depth of water when the tank is not rotating, and $a$ is the radius of the tank.

For the equations of motion:

$$
\begin{aligned}
-2 \omega v_{\phi} & =-g \frac{d \eta}{d r}+\nu \frac{\partial^{2} v_{r}}{\partial z^{2}} \\
2 \omega v_{r} & =\quad v \frac{\partial^{2} v_{\phi}}{\partial z^{2}}
\end{aligned}
$$

where $v_{r}$ and $v_{\phi}$ are the respective components of velocity relative to the tank, $\eta$ is the perturbation in the height of the free surface, and $v$ is the kinematic viscosity.

These equations assume: (a) steady flow with negligibly small inertial terms, (b) hydrostatic equilibrium in the vertical direction, (c) axial symmetry, i.e. no $\phi$ dependence of $\vec{v}$ or $\eta$, (d) negligible lateral friction, i.e. negligible contribution from the lateral NavierStokes term $\nu \nabla_{H}^{2} \vec{v}$ where $\nabla_{H}^{2}$ is the horizontal Laplacian. (As will be shown subsequently, $\boldsymbol{\nu} \nabla_{H}^{2} \vec{v}$ turns out to be identically zero for the solution obtained, which is therefore in reality a complete solution for the linearized NavierStokes equation in this case).

The vertical boundary conditions are

$$
v_{r}=v_{\phi}=0
$$

along $z=0$, the bottom of the tank, and

$$
\frac{\partial v_{r}}{\partial z}=\frac{\partial v_{\phi}}{\partial z}=0
$$

along $z=h$, the free surface.

The quantity $\frac{d \eta}{d r}$ is carried as a parameter and is ultimately determined by the source and sink conditions at $r=0$ and $r=a$ and the requirements of continuity.

It is convenient to introduce the notation

$$
d \equiv\left(\frac{\nu}{\omega}\right)^{\frac{1}{2}} ; \mu \equiv \frac{h}{d} ; \quad \zeta \equiv \frac{z}{d}
$$

The solutions of equations (2), (3), (4), (5) are then found by standard methods:

$$
\begin{gathered}
v_{r}=-\frac{g}{2 \omega} \frac{d \eta}{d r} \\
\cdot\left[\frac{\sinh (2 \mu-\zeta) \sin \zeta+\sin (2 \mu-\zeta) \sinh \zeta}{\cosh 2 \mu+\cos 2 \mu}\right] \text { (6) } \\
v_{\phi}=\frac{g}{2 \omega} \frac{d \eta}{d r} \\
\cdot\left[\mathrm{I}-\frac{\cosh (2 \mu-\zeta) \cos \zeta+\cos (2 \mu-\zeta) \cosh \zeta}{\cosh 2 \mu+\cos 2 \mu}\right]
\end{gathered}
$$

In these experiments $\mu$ is of the order of to to 50 , and equations (6) and (7) reduce to the 
simpler forms with the familiar laminar $\mathrm{Ek}$ man spiral in the neighborhood of the bottom of the tank:

$$
\begin{aligned}
& v_{r}=-\frac{g}{2 \omega} \frac{d \eta}{d r} \varepsilon^{-\zeta} \sin \zeta \\
& v_{\phi}=\frac{g}{2 \omega} \frac{d \eta}{d r}\left[1-\varepsilon^{-\zeta} \cos \zeta\right]
\end{aligned}
$$

The vertically integrated radial and zonal volume transports $V_{r}$ and $V_{\phi}$ become, respectively:

$$
\begin{aligned}
& V_{r}=-\frac{g}{2 \omega} \frac{d \eta}{d r} \frac{d}{2} \mathrm{~cm}^{3} / \mathrm{sec}_{.}, \mathrm{cm} \\
& V_{\phi}=\frac{g h}{2 \omega} \frac{d \eta}{d r}\left[\mathrm{I}-\frac{d}{2 h}\right] \mathrm{cm}^{3} / \mathrm{sec} ., \mathrm{cm}
\end{aligned}
$$

For particular cases, $\frac{d \eta}{d r}$ is determined by continuity and source-sink conditions:

(a) Source of $S_{0} \mathrm{~cm}^{3} / \mathrm{sec}$. at $r=0$, and sink of equal intensity uniformly distributed around the periphery at $r=a$. Free surface neither rises nor falls, i.e. vertical velocity $\dot{\eta}_{0}=0$. Continuity requires:

$$
2 \pi r V_{r}=S_{0}
$$

and combination of equations (IO) and (I2) gives

then

$$
\frac{d \eta}{d r}=-\frac{2 \omega S_{0}}{\pi g d r}
$$

$$
\begin{aligned}
& V_{r}=-\frac{S_{0}}{2 \pi r} \\
& V_{\phi}=-\frac{h S_{0}}{\pi d} \frac{\mathrm{I}}{r}\left[\mathrm{I}-\frac{d}{2 h}\right]
\end{aligned}
$$

and $v_{\phi}$ becomes essentially a $\mathbf{I} / r$ vortex above the Ekman layer.

(b) If the surface is allowed to rise or fall (i.e. the basin fill or empty because of the sourcesink distribution), $\dot{\eta}_{0} \neq 0$, and the equation of continuity requires:

$$
\frac{\mathrm{I}}{r} \frac{\partial\left(r V_{r}\right)}{\partial r}=-\dot{\eta}_{0}
$$

Combination of (I6) and (Io) and integration give:

$$
\frac{d \eta}{d r}=\frac{2 \omega \dot{\eta}_{0}}{g d} r+\frac{C}{r}
$$

where $\mathrm{C}$ is the constant of integration.

(c) If a source $S_{0}$ is uniformly distributed around the periphery, and $V_{r}=0$ at $r=0$, we have:

$$
\begin{aligned}
\dot{\eta}_{0} & =\frac{S_{0}}{\pi a^{2}} ; C=0 \\
V_{r} & =-\frac{S_{0}}{2 \pi a^{2}} r \\
V_{\phi} & =\frac{S_{0}}{\pi a^{2}} \frac{h}{d}\left[\mathrm{I}-\frac{d}{2 h}\right] r
\end{aligned}
$$

Here $v_{\phi}$ represents a "solid rotation" above the Ekman layer. $v_{\phi}$ is cyclonic and the Ekman transport, $V_{r}$, is radially inward for positive $S_{0}$.

(d) If there is a point source $S_{0}$ at $r=0$ and $V_{r}=\mathrm{o}$ at $r=a$, we have:

$$
\begin{aligned}
C & =-\frac{2 \omega S_{0}}{\pi g d} \\
V_{r} & =\frac{S_{0}}{2 \pi a}\left(\frac{a}{r}-\frac{r}{a}\right) \\
v_{r} & =\frac{S_{0}}{\pi a d}\left(\frac{a}{r}-\frac{r}{a}\right) \varepsilon^{-\zeta} \sin \zeta \\
V_{\phi} & =-\frac{S_{0} h}{\pi a d}\left(\frac{a}{r}-\frac{r}{a}\right)\left[\mathrm{I}-\frac{d}{2 h}\right] \\
v_{\phi} & =-\frac{S_{0}}{\pi a d}\left(\frac{a}{r}-\frac{r}{a}\right)\left[\mathrm{I}-\varepsilon^{-\zeta} \cos \zeta\right]
\end{aligned}
$$

Here $v_{\phi}$ above the Ekman layer is determined by the superposition of a I/ $r$ vortex and a solid rotation in the opposite sense in such a way as to give $v_{\phi}=0$ at $r=a$.

One experiment has been performed with a peripherally distributed source (case (c)) to verify the prediction of eq. (I9) that the zonal flow will be a solid rotation. A solid rotation was observed under these circumstances.

All the obscrvations of instability of the Ekman flow were made with a central source (case (d)) for the situation described by eqs. (20)-(23). The physical interpretation or description of this situation is as follows: The presence of a central source (positive $S_{0}$ ) must 
produce a uniform vertical velocity throughout the fluid as the surface rises. Water must be transported radially outward from the central source, and such transport can occur only in the frictional boundary layer. The source therefore induces a general anticyclonic zonal geostrophic flow with an Ekman layer at the bottom. A net cross-isobar transport of water radially outward (to the left of the zonal flow) takes place in the Ekman layer, the zonal geostrophic velocities adjusting themselves to produce the required transport and horizontal divergence in the Ekman layer.

If the equations of motion were written to include the effect of lateral friction, eq. (2) would have on the right hand side the added term

$$
v\left[\frac{\mathrm{I}}{r} \frac{\partial}{\partial r}\left(r \frac{\partial v_{r}}{\partial r}\right)-\frac{v_{r}}{r^{2}}\right]
$$

and eq. (3) would have a similar one in $v_{\phi}$. Solutions with radial dependence of the form $r$ or $\mathrm{I} / r$ make this term identically zero. Thus the basic field solutions developed above are actually solutions to the complete, linearized $\mathrm{Na}$ vier-Stokes equations applicable to this problem.

\section{Quantitative verification of the basic flow}

The basic flow as described by eqs. (20)(23) was verified quantitatively in two ways:

(a) by direct observation of the zonal surface velocity $\nu_{\phi}$ for various values of $\omega$ and $S_{0}$.

(b) by direct observation of the rate of radial spread of the colored Ekman layer when dye was introduced into the source.

A comparison of observed and predicted zonal surface velocities is shown in Figure 3. Each set of points, denoted by a particular symbol, represents the results at different radii for a particular run at some value of $\omega, \nu$, and $S_{0}$. Instability of the Ekman layer was present in each run. Under the existing circumstances, however, the scatter of the data is within the range of experimental uncertainty. We are led to say that in these experiments the zonal surface velocities agree, within experimental error, with the predicted basic field values, and it is not possible to assert a significant effect of the Ekman instability on the basic flow.

A similar result is obtained for observations of the radial spread of the Ekman layer. AssuTellus XIII (1961). 1

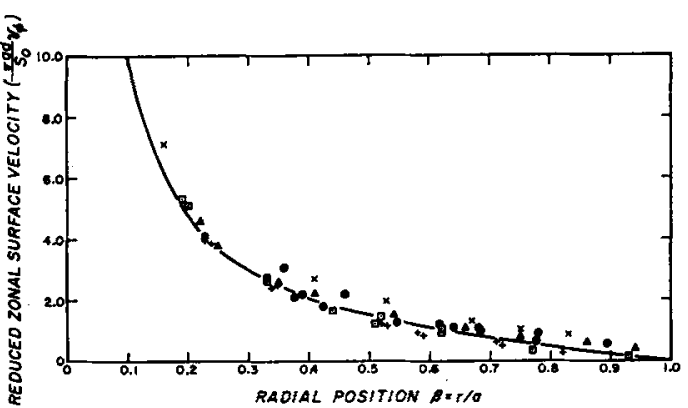

Fig. 3. Scaled zonal velocity $v_{\phi}$ vs. radial position, for various combinations of source strength and angular velocity.

Theoretical curve: $-\frac{\Pi_{a d}}{S_{0}} v_{\phi}=\frac{1}{\beta}-\beta$

Experimental Runs

$\begin{array}{ccc}\text { Symbol } & \begin{array}{c}S_{0} \\ \left(\mathrm{~cm}^{3} / \mathrm{sec}\right)\end{array} & \begin{array}{c}\omega \\ \text { radians/sec })\end{array} \\ \bigcirc & 2.71 & 1.02 \\ \times & 3.32 & 2.01 \\ - & 3.39 & 1.02 \\ \triangle & 3.30 & 2.01 \\ \square & 3.65 & 2.02 \\ + & 3.74 & 1.00\end{array}$

ming that the visible radial spread will be determined by the maximum value of $\nu_{r}$ in the Ekman layer, eq. (2I) gives:

$$
v_{r \max }=\frac{0.322 S_{0}}{\pi a d}\left(\frac{\mathrm{I}}{\beta}-\beta\right)
$$

where $\beta \equiv r / a$. Integration of eq. (24) gives:

$$
t=\mathrm{I} .553 \frac{\pi a^{2} d}{S_{0}} \ln \left(\mathrm{I}-\beta^{2}\right)
$$

where $t=0$ when $r=0$, and $t$ is the time taken for a particle to reach radial position $\beta$.

Figure 4 shows a scmilogarithmic plot of $\left(I-\beta^{2}\right)$ vs. $t$. Only one run of this kind was made, and the scatter of results from run to run is therefore not established, but the observed values are considered to be in good agreement with predictions, especially at values of $\beta$ of about 0.3 to 0.5 where the instabilities and their critical limit were usually observed. The uncertainty indicated for the experimental points in Figure 4 stems from the fact that the outer boundary of the colored ring was not perfectly circular.

We thus conclude that the basic flow field obtained in these experiments is in good agree- 


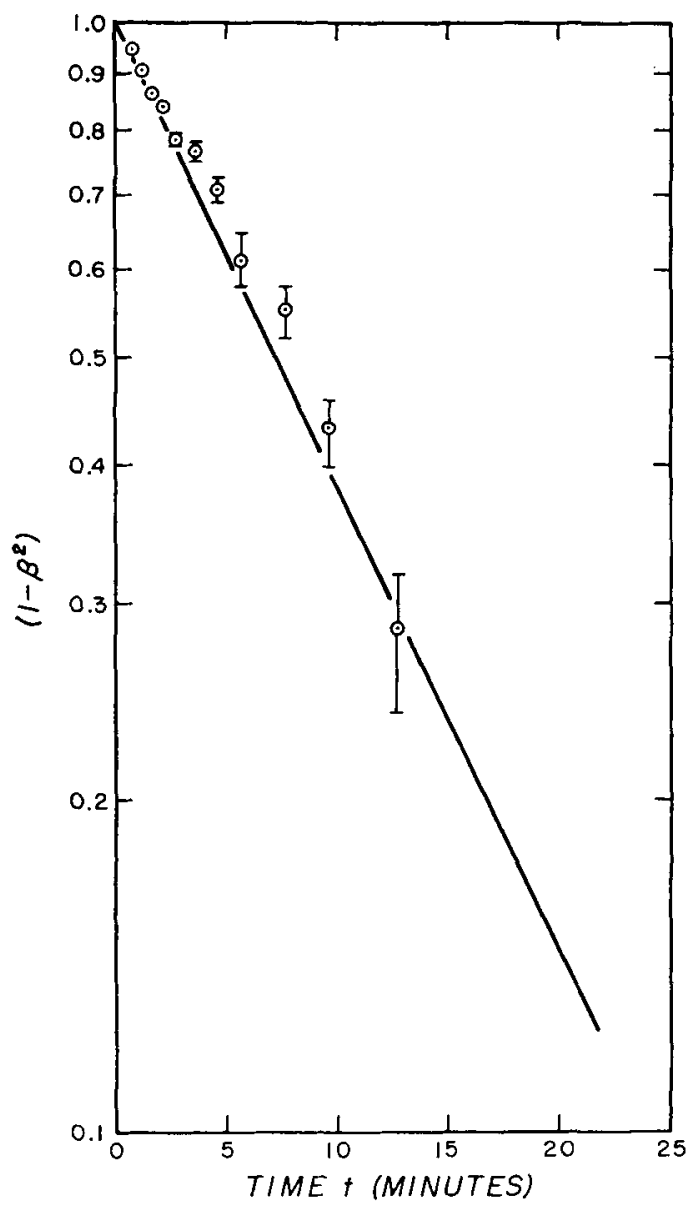

Fig. 4. Radial spread of Ekman laycr vs, time

Theoretical curve: eq. (25)

Experimental observations:

$$
\begin{aligned}
& \omega=1.79 \mathrm{radians} / \mathrm{sec} \text {; } S_{0}=2.42 \mathrm{~cm}^{3} / \mathrm{sec} . \\
& a=65 \mathrm{~cm} .
\end{aligned}
$$

Range of uncertainty indicates out of roundness of spreading Ekman layer.

ment with the values predicted by the analysis of the preceding scction. Noticeable departures from simple laminar flow are, however, invariably obscrved in two regions: (I) a turbulent region within a few centimeters of the center of the tank (2) a turbulent band several centimeters wide at the outer rim. In these regions the laminar flow is unstable, and turbulence penetrates the entirc depth of fluid. Apparently the presence of these two turbulent regions does not scriously affect the basic flow regime in the intcrior of the basin.

\section{Observed instability of the Ekman flow}

Figures $I$ and 2 show typical top and side vicws, respectively, of the curtains of fluid which originate in the Ekman layer and penctrate the entire depth of flow, The phenomenon is characterized by a "wave length", $\lambda$, or spacing between the curtains, of the order of I to $2 \mathrm{~cm}$. and by a critical radius $r_{c}$ beyond which the curtains do not occur. All the accumulated values of $\lambda$ and $r_{c}$ for various combinations of $S_{0}, \omega, h$, and are $v$ summarized in Table I together with accompanying values of Ekman, Taylor, Reynolds, Rossby, and Froude numbers. The specific definitions for the various numbers in the present context are indicated in Table I.

STERN's (1960) assymptotic analysis in Cartesian coordinates predicts an instability of essentially the type obscrved in these experiments-a flow drawing its energy from the Ekman layer and penctrating the entire depth of fluid. He further predicts that the wave length of the resulting cclls will be a function of the Taylor number, $E$ (sce Table I):

$$
\frac{\lambda}{d} \sim 6 E^{b}
$$

Figure 5 shows a plot of observed values of

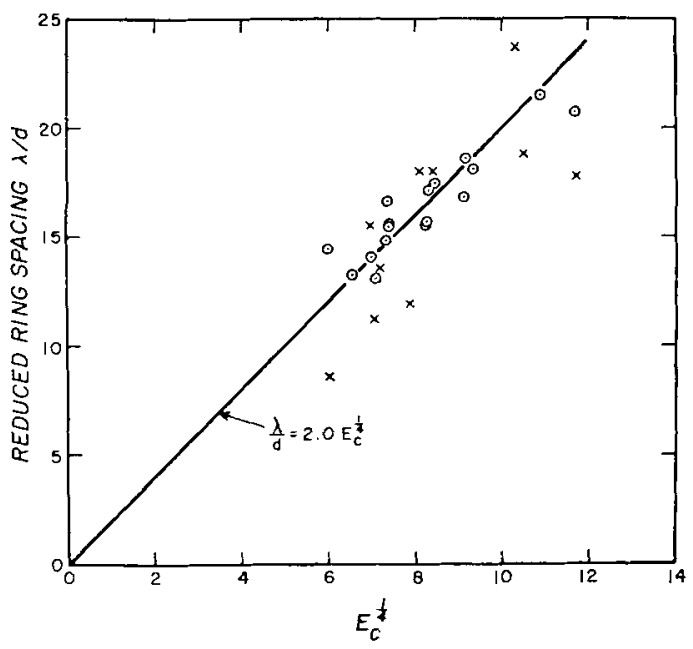

Fig. 5. Reduced ring spacing, $\lambda / d$ vs. fourth root of Taylor number, $E_{c}^{1 / 4}$.

Points from Table I for cases in which $r_{c}$ was reasonably wcll defined.

$\times$ Points marked (?) in Table I for cases in which $r_{c}$ was very poorly defined. 
Table 1. Observed ring spacing and critical radii.

\begin{tabular}{|c|c|c|c|c|c|c|c|c|c|c|c|c|c|}
\hline \multirow{2}{*}{$\begin{array}{l}\text { Angular } \\
\text { Velocity } \\
\qquad \omega \\
\mathrm{rad} / \mathrm{sec} .\end{array}$} & \multirow{2}{*}{$\begin{array}{c}\text { Source } \\
\text { Strength } \\
S_{0} \\
\mathrm{~cm}^{3} / \mathrm{sec} .\end{array}$} & \multirow{2}{*}{$\begin{array}{c}\text { Kine- } \\
\text { matic } \\
\text { Viscosity } \\
v \times 10^{2} \\
\mathrm{~cm}^{2} / \mathrm{sec}\end{array}$} & \multirow{2}{*}{$\begin{array}{c}\text { Average } \\
\text { Ring } \\
\text { Spacing } \\
\lambda \\
\mathrm{cm} .\end{array}$} & \multirow{2}{*}{$\begin{array}{c}\text { Critical } \\
\text { Radius } \\
r_{c} \\
\\
\mathrm{~cm} .\end{array}$} & \multirow{2}{*}{$\begin{array}{c}\text { Depth } \\
\text { At } \boldsymbol{r}_{c} \\
h_{c} \\
\mathrm{~cm} .\end{array}$} & \multirow{2}{*}{$\begin{array}{c}\text { Reduced } \\
\text { Ring } \\
\text { Spacing } \\
\lambda / d\end{array}$} & \multicolumn{7}{|c|}{$\begin{array}{l}\text { Dimensionless Parameters } \\
\text { Applicable to flow at } r_{c}\end{array}$} \\
\hline & & & & & & & $E_{e}$ & $R, c$ & $R_{I C}$ & $\varepsilon_{e}$ & $F_{c}$ & $R_{C} \times \mathrm{IO}^{3}$ & $R_{E(19)}$ \\
\hline 2.18 & 2.87 & .983 & 0.87 & 27 & $3.4^{\circ}$ & I 3.0 & 2570 & 2.80 & I 4 I & .0197 & I. 04 & 7.0 & $4 \cdot 4^{8}$ \\
\hline 2.18 & 2.77 & .971 & I. 38 & 38 & 9.20 & 20.7 & I 9000 & I. 59 & 221 & .0072 & $0.7^{6}$ & 2.8 & $4 \cdot 37$ \\
\hline 2.18 & 2.82 & .915 & 1.20 & 29 & $5.4^{\circ}$ & I8.6 & 6940 & 2.70 & 225 & .0120 & 0.75 & 6.0 & $4 \cdot 73$ \\
\hline 2.18 & 2.83 & .983 & $1.2 I$ & 28 & $4 \cdot 4^{\circ}$ & I 8.0 & $433^{\circ}$ & 2.65 & I74 & .0152 & 0.86 & 6.3 & $4 \cdot 42$ \\
\hline I. 57 & 2.83 & .925 & I. 65 & $34(?)$ & 9.15 & $2 I .5$ & I 4200 & 2.06 & 245 & $.008_{4}$ & 0.32 & 4.6 & $4 \cdot 70$ \\
\hline I. 57 & 2.80 & .947 & I. 40 & 32 & $5 \cdot 45$ & I 8.0 & 4890 & 2.24 & I 57 & .0143 & $0.4^{8}$ & $5 \cdot 5$ & $4 \cdot 54$ \\
\hline 2.18 & $2.7^{6}$ & I. I $4^{O}$ & 1.20 & 22 & 3.90 & 16.6 & 2920 & 3.10 & I 68 & .0185 & 0.60 & 10.2 & $3 \cdot 7 \mathrm{I}$ \\
\hline 2.18 & 2.76 & .893 & I.O9 & 28 & $4 \cdot 4^{\circ}$ & I 7.1 & $475^{\circ}$ & $2.9 \mathrm{I}$ & $20 I$ & .0145 & 0.86 & 6.6 & $4 \cdot 74$ \\
\hline I. 57 & 2.85 & .947 & $1.4^{\circ}$ & $27(?)$ & 5.05 & I 8.0 & 4210 & 2.89 & I 88 & .0154 & .037 & 8.3 & 4.62 \\
\hline I. 92 & $2.7^{6}$ & .900 & I. 20 & 25 & 4.90 & 17.5 & 5110 & $3.4^{\circ}$ & 243 & .0140 & $0.4^{8}$ & 9.3 & $4 \cdot 7^{\circ}$ \\
\hline I. 64 & 2.75 & .906 & I. 40 & $5 \circ(?)$ & 8.20 & 18.8 & I 2100 & 0.80 & 89 & $.009 \mathrm{I}$ & 0.84 & I. 2 & 4.65 \\
\hline 2.18 & 2.82 & $.90 I$ & 0.70 & $30(?)$ & 4.05 & 10.9 & 3960 & 2.62 & 165 & . OI 59 & I.07 & 5.6 & 4.80 \\
\hline I.92 & $2.8 I$ & .932 & I. 08 & 24 & 3.80 & I 5.5 & 2980 & $3 \cdot 4^{6}$ & 189 & .0183 & 0.57 & 10.0 & 4.63 \\
\hline I.O4 & $2.7^{8}$ & I. I 30 & I. 50 & 19 & 3.75 & I 4.4 & I 290 & 3.65 & I3I & .0278 & 0.1 I & 20.0 & 3.77 \\
\hline 2.18 & 2.87 & .990 & 1.20 & $44(?)$ & 9.20 & 17.8 & I 8800 & I. IO & I $5 \mathrm{I}$ & .0073 & I.02 & I. 7 & $4 \cdot 45$ \\
\hline I. $0_{4}$ & 2.85 & .893 & I. 20 & 32 & 3.90 & I 3.2 & I 840 & 2.37 & 102 & .0233 & 0.29 & 6.7 & 4.90 \\
\hline I. $3^{2}$ & $2.8 \mathrm{I}$ & .902 & I.I 6 & 32 & 4.05 & 14.0 & 2400 & 2.35 & I 15 & .0204 & 0.45 & 6.1 & $4 \cdot 78$ \\
\hline I. 64 & 2.80 & .893 & I. I 2 & 29 & 4.15 & I 5.2 & 3160 & 2.75 & I 54 & .0178 & 0.56 & 7.0 & $4.8 \mathrm{I}$ \\
\hline I. 64 & 2.13 & .904 & I.10 & 28 & 4.00 & I 4.8 & 2920 & 2.20 & I I 9 & .0185 & 0.54 & 5.8 & 3.62 \\
\hline 1.64 & I. 52 & .897 & I. I 6 & 27 & 4.05 & I 5.7 & 3020 & I. 67 & 92 & .0182 & 0.49 & 4.6 & \\
\hline I. 64 & 3.52 & .897 & I. I 6 & 34 & 5.05 & I 5.7 & 4630 & 2.67 & I 8 I & .0147 & 0.63 & 7.8 & 6.02 \\
\hline I. 64 & 4.25 & .913 & I. I 6 & 36 & 5.05 & I 5.5 & 4570 & 2.85 & 193 & .0148 & $0.7^{\circ}$ & 5.9 & 7.14 \\
\hline I.04 & 2.67 & I. 165 & $0.9(?)$ & $28(?)$ & 3.85 & 8.5 & 1320 & 2.I 3 & 77 & .0275 & 0.22 & 8.0 & 3.52 \\
\hline 1.04 & 2.75 & I.205 & I.2(?) & $26(?)$ & 5.10 & I I. 2 & 2240 & 2.33 & I IO & $.02 \mathrm{I} \mathrm{I}$ & 0.15 & 9.6 & 3.50 \\
\hline 2.18 & 2.73 & I. 205 & I.O(?) & $20(?)$ & 3.85 & I 3.5 & 2680 & 3.25 & 168 & .0193 & $0.5^{\circ}$ & I2.I & 3.47 \\
\hline I.00 & 4.00 & I.060 & I. 6 & $27(?)$ & 5.05 & I 5.5 & 2400 & 3.71 & 182 & .0204 & o. I 5 & I 4.2 & 5.80 \\
\hline I. 25 & 4.90 & .960 & 2.I (?) & $50(?)$ & $9 \cdot 30$ & 23.7 & I I 300 & $1 \cdot 3^{2}$ & 140 & .0094 & 0.43 & $2 \cdot 3$ & $7 \cdot 83$ \\
\hline 2.18 & 2.65 & $.8 \mathrm{Io}$ & I.I & 33 & 5.27 & I 8.1 & 7560 & 2.49 & 216 & .0115 & 1.00 & 3.0 & 5.02 \\
\hline 2.18 & 2.82 & .716 & 0.96 & 30 & $4 \cdot 7^{6}$ & I6.8 & 6940 & 3.23 & 269 & .0120 & 10.92 & 6.2 & 6.04 \\
\hline
\end{tabular}

Dimensionless parameters at $\mathbf{r}_{\boldsymbol{c}}$ :

Taylor no. $\quad E_{\iota} \equiv \frac{\omega h_{c}^{2}}{\nu}$

Ekman no. $\quad \varepsilon_{c} \equiv \frac{\mathbf{I}}{h_{c}}\left(\frac{\mathrm{v}}{\omega}\right)^{\frac{1}{2}}$

Reynolds no. for Ekman layer:

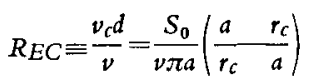

$R_{E(19)}=$ Reynolds no. at $r=19 \mathrm{~cm}$.

$\frac{\lambda}{d}$ versus $E_{c}^{1 / 4}$, taken from Table I. (The local Taylor number of the flow varies with $r$, but $\lambda$ is uniform within a few percent in the region where the rings occur. It seems reasonable to take as the characteristic Taylor number for any given case that value which obtains at the critical radius $r_{c}$ ).

We do not have enough data or attempts at identical repetition of experiments to state posTellus XIII (1961), 1
Reynolds no. for interior flow:

$$
R_{\mathrm{IC}} \equiv \frac{\nu_{c} h_{c}}{\nu}=\frac{R_{E C}}{\varepsilon_{c}}
$$

Froude no. $F_{c} \equiv \frac{\left(r_{c} \omega\right)^{2}}{g h_{c}}$

Rossby no. $R_{c}=\frac{\nu_{c}}{r_{c} \omega}=\frac{R_{E C}}{r_{c}}\left(\frac{\nu}{\omega}\right)^{\frac{1}{2}}$

(?) denotes poorly defined values

itively whether the scatter of points in Figure 5 contains systematic dependence on other parameters or represents the intrinsic variability of the observations. Assuming the latter, the observations scatter about a straight line represented approximately by

$$
\frac{\lambda}{d}=2.0 E_{c}^{1 / 4}
$$

This is consistent with Stern's asymptotic 


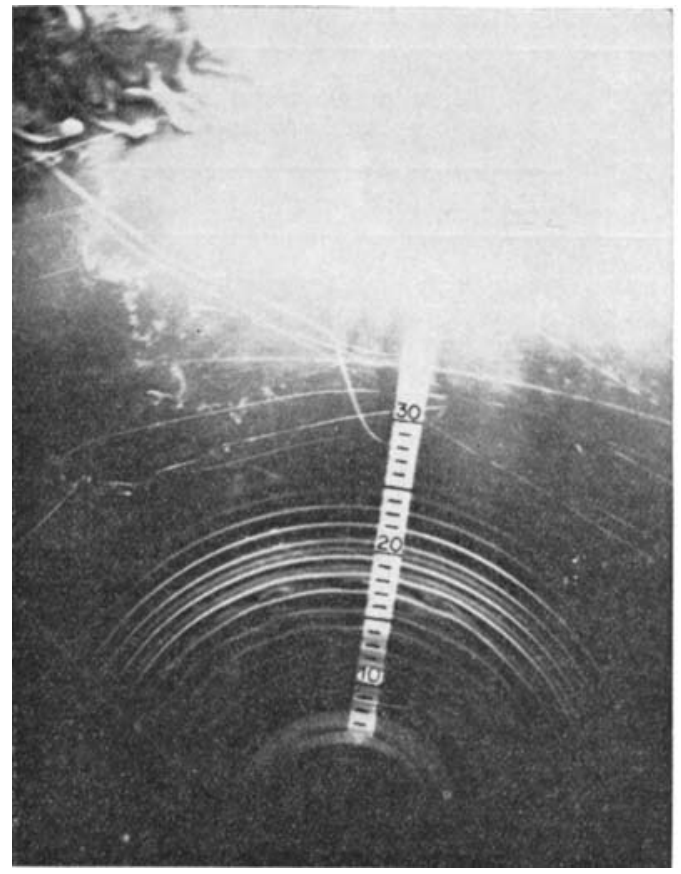

Fig. 6. Downward circulation between rising curtains: Intense rings are the rising jets; fainter rings at 19 and 20 $\mathrm{cm}$. marks, between the more intense rings, mark downward circulation of dye shortly after it reached the water surface. Marks of downward circulation between rings closer to center have become more diffuse since dye reached these regions several minutes before instant of photograph. Upper left hand corner of photograph shows band of turbulence along outer rim of tank.

result for the simpler gcometry. Two Rcynolds numbers at the critical radius $r_{c}$ have been tabulated in Table I. The Reynolds number $R_{I C}$ of the intcrior geostrophic flow varics widcly and shows no disccrnable correlation with other parameters. The Reynolds number $R_{E C}$ of the Ekman flow, when questionable values of $r_{c}$ are omitted, lies in the relatively narrow range between I.6 and 3.6, (the extremc range of values of this number for various radial positions in these cxperiments is of the order of o to ro). The apparent corrclation of the onset of rings with a narrow range of values of $R_{E C}$ implies that the instability draws its encrgy from the Ekman layer rather than from the geostrophic flow, and this view is also consistent with Stern's analysis.

The last column in Table I gives values of the Reynolds number of the Ekman layer at a radius of $19 \mathrm{~cm}$, which was usually about the mid-region for the occurrence of the vertical sheets. Comparison of values of $R_{E(19)}$ with $R_{E C}$ will indicate the extent to which the Reynolds number at the critical radius is excecded in the interior of the region of instability; this might be an index to the importance of finite amplitude effects in this region.

In general, downward circulation between the rising curtains was considerably less sharp and intense than that of the rising phase. A top view, showing evidence of the downward motions, is shown in Figure 6. The fainter rings, when observed from the side of the tank, were seen to be moving downward, and do not represent a rising mode of smaller wavelength. Both upward and downward motions were also observed when dye was injected very slowly from capillary probes inserted at various points in the body of the flow.

The upward velocities of the curtains are roughly $0.7 \mathrm{~cm} / \mathrm{min}$., i.e. an order of magnitude of about I \% of the basic zonal velocity.

On some occasions a fairly regular, small scale, zonal wave or vortex structure was formed on the rising curtains. An illustration of this effect is shown in Figure 7. This phenomenon did not scem to correlate with systematically outlying values of either of the calculated Reynolds numbers; in a substantial number of cases the waves did not form in experiments conducted at Reynolds numbers higher than those in which waves were observed. The waves appeared to occur somewhat more frequently however in experiments at

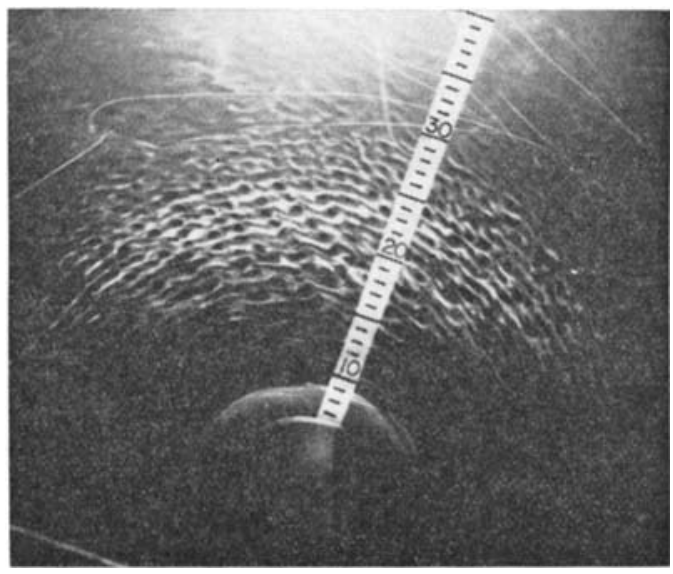

Fig. 7. Illustration of small scale zonal disturbances which were occasionally observed. Although these waves could not be unequivocally correlated with relatively high Reynolds numbers, they seemed to occur somewhat more frequently at high source rates and low viscosities. 
higher source strengths or at lower viscosities. No rings or curtains were observed to form in several experiments performed at temperatures below $13^{\circ} \mathrm{C}$., i.e. at kinematic viscosities greater than about $0.012 \mathrm{~cm}^{2} / \mathrm{sec}$.; in these cases the Ekman flow was laminar and stable.

Since the ranges of variation of $\omega$ and $h$ are limited by the design of the existing equipment, it has not been possible to vary these parameters over a wide enough range to test the expectation that the cut-off of the ring phenomenon depends on a dimensionless parameter and is not determined solely by the higher viscosity of the water at the lower temperatures Every effort, however, has been made to perform the experiments under conditions of temperature homogenity throughout the tank and at temperature equilibrium between the tank and the room. The tank was carefully covered to minimize both wind and evaporation effects. The experiments were performed in the winter time, and when low temperature runs were underway, the room itself was cooled to the temperature of the run by turning off the heat. If any error in maintainance of inhomogeneity existed, it was more probably in the direction of allowing the existence of vertical stability in the body of water in the tank.

In a few instances, rings did not form under conditions in which they normally occurred. Disturbances such as jarring the tank and briefly interrupting the source flow then led to the formation of rings-sometimes in competing sets at different radii, the competing sets then shifted and wandered somewhat until they interlocked into a normal pattern.

\section{REFERENCES}

Stern, M. E., I960; Instability of Ekman Flows at Large Taylor Numbers. Tellus 12, pp. 399-4I7.

Stommel, H., Arons, A. B.; Faller, A. J.; 1958: Some Examples of Stationary Planetary Flow Patterns in Bounded Basins. Tellus 10, pp. 179-187. 